\title{
Graphene Nucleation Density on Copper: Fundamental Role of Background Pressure
}

Ivan Vlassiouk, ${ }^{*}$ Sergei Smirnov, ${ }^{\neq *}$ Sumedh P. Surwade, ${ }^{\dagger}$ Murari Regmi, ${ }^{\dagger}$ Nishtha Srivastava, ${ }^{\S}$ Randall Feenstra, ${ }^{\S}$ Gyula Eres, ${ }^{\dagger}$ Chad Parish, ${ }^{\dagger}$ Nick Lavrik, ${ }^{\dagger}$ Panos Datskos, ${ }^{\dagger}$ Sheng Dai ${ }^{\dagger}$ and Pasquale Fulvio ${ }^{\dagger}$

$\uparrow$ Oak Ridge National Laboratory, Oak Ridge, TN

$\$$ Department of Chemistry and Biochemistry, New Mexico State University, Las Cruces, NM

$\S$ Department of Physics, Carnegie Mellon University, Pittsburgh, PA

ABSTRACT In this paper we discuss the effect of background pressure and synthesis temperature on the graphene crystal sizes in chemical vapor deposition (CVD) on copper catalyst. For the first time, we quantitatively demonstrate a fundamental role of the background pressure and provide the activation energy for graphene nucleation in atmospheric pressure CVD $(9 \mathrm{eV})$, which is substantially higher than for low pressure CVD $(4 \mathrm{eV})$. We attribute the difference to a greater importance of copper sublimation in low pressure CVD, where severe copper evaporation likely dictates the desorption rate of active carbon from the surface. At atmospheric pressure, where copper evaporation is suppressed, the activation energy is assigned to the desorption energy of carbon clusters instead. The highest possible temperature, close to the melting point of copper, should be used for large single crystal graphene synthesis. Using these conditions, we have synthesized graphene single crystals approaching $1 \mathrm{~mm}$ in size. Single crystal nature of synthesized graphene was confirmed by low energy electron diffraction. We also demonstrate that CVD of graphene at temperatures below $1000 \mathrm{oC}$ shows higher nucleation density on (111) than on (100) and (101) copper surfaces but there is no identifiable preference at higher temperatures.

\section{Introduction.}

Demonstration of graphene growth on copper [1] by chemical vapor deposition and further extension of this synthesis to industrial scale [2-4] resulted in a great leap toward the understanding of the growth mechanisms. To date, the graphene films prepared by CVD on copper foils have polycrystalline structure, which is governed by the nucleation density and the growth kinetics. The nucleation density of graphene during the growth process is of fundamental importance since it determines the graphene domain sizes, which, in turn, plays a crucial role in the material quality.[5,6] In fact, the size domains and interconnections between them determine material properties such as mechanical strength, charge carrier mobilities, doping levels, and thermal conductivity.[7] Therefore, a clear understanding of the parameters affecting the nucleation density of graphene is needed for the ultimate control over the material characteristics.

Recent studies focused on decreasing the nucleation density of graphene on metal catalyst surfaces resulted in the recipes for synthesis of large single crystalline graphene grains. For example, large grains were grown on catalysts with negligible carbon solubility such as copper,[8-13] as well as, on those with larger carbon solubility such as platinum [14] using both the low and atmospheric pressure CVD processes.[15] These growth recipes, however, did not provide detailed explanations to ensure the ultimate control of graphene nucleation densities. For instance, large graphene grains were grown on copper foils folded into "pockets", where it was believed to "improve" [13] the growth atmosphere. Others pointed to a necessity of longer 
catalyst annealing time [11] presumably decreasing the number of surface defects suitable for graphene nucleation. Effects of other growth parameters such as carbon precursor and hydrogen cocatalyst partial pressures [8], growth temperature on the graphene grain sizes were investigated qualitatively as well.[16,20] For example, Li et al.[16] showed the importance of temperature and methane flux in controlling the graphene domain size. In particular, the growth temperature and pressure in the reactor affect every step of the growth process, from the catalyst annealing to the formation of active carbon species, carbon diffusion on the catalyst surface, kinetics and thermodynamics of various chemical reaction steps between carbon intermediates and the metal substrate, formation of stable graphene nuclei and subsequent crystal growth.

Here we focus on more quantitative analysis of temperature effects in graphene growth on copper. Namely, we elucidate its effect on the graphene nucleation density and the terminal graphene domain size in both, atmospheric pressure and low pressure CVD (APCVD and LPCVD). We discuss the role of background pressure in a CVD reactor on graphene synthesis, and elucidate the necessary conditions for synthesis of large graphene domains approaching 0.7 $\mathrm{mm}$ in size.

\section{Results}

Graphene growth from hydrocarbon precursor, such as methane used in this study, requires access to catalytic copper surface by hydrocarbon and hydrogen cocatalyst that helps producing activated carbon from the adsorbed hydrocarbon. Thus the rate of growth declines when graphene continues to cover greater and greater portion of the copper surface. Moreover, as we have demonstrated before,[20] hydrogen's role is dual and includes etching weak C-C and C-H bonds. This causes further slowing down the rate of growth at high graphene coverage and ultimate survival of the more stable zigzag edges, especially at high hydrogen concentrations. The resulting graphene domains at high hydrogen concentrations accept hexagonal shape with zigzag edges.[20] If the amount of carbon source is low, the domains can stop growing upon reaching a terminal size, when the density of nucleation is low as well. At such a point, the rates of growth and etching become equal. Such oversimplified general equilibrium is represented by reaction 1.

$$
\text { Graphene }+\mathrm{C}_{\mathrm{x}} \mathrm{H}_{\mathrm{y}}(\mathrm{s}) \Leftrightarrow\left(\text { Graphene- } \mathrm{C}_{\mathrm{x}}\right)+\mathrm{yH}(\mathrm{s})
$$

\section{Atmospheric pressure CVD (APCVD)}

First, we focus on the atmospheric pressure CVD (APCVD) and chose the conditions close to those for terminal coverage: $2 \mathrm{hr}$ deposition with 19 Torr of hydrogen and $10.5 \mathrm{mTorr}$ of methane, which produce hexagonal shape of graphene domains. Prior to deposition, $\mathrm{Cu}$ foils were polished in phosphoric acid and annealed for half an hour in the same atmosphere but without methane. Both of these treatments were shown to be crucial for minimizing nucleation sites due to impurities and roughness.[4,20]

Figure 1 (A and B) shows the nucleation density and graphene grain size evolution for APCVD as a function of the growth temperature while all other experimental parameters are kept unchanged. Suitable temperature range under these experimental conditions allows unambiguously recognizable hexagonal crystals by SEM at temperatures from 950 to $1080{ }^{\circ} \mathrm{C}$. Analysis of the data reveals that the graphene nucleation density strongly declines upon increasing temperature with the effective (negative) activation energy of $E_{n u c}=9 \mathrm{eV}$. Such a large activation barrier results in almost five orders of magnitude change in the nucleation 
density in a narrow $130{ }^{\circ} \mathrm{C}$ temperature range (Figure 1C). We should emphasize that, because of electropolishing and annealing prior to deposition, this dependence is not compromised by variation of roughness or impurities (except for $<0.2 \%$ of $\mathrm{Cu}$ impurity). Indeed, annealing of foils at the deposition temperature or at a higher temperature produces similar results; additional experiments showed minor difference if annealing was performed at $1080{ }^{\circ} \mathrm{C}$. The largest variation is observed at the lowest deposition temperature $\left(950^{\circ} \mathrm{C}\right.$, blue point in Figure $\left.1 \mathrm{C}\right)$. Nucleation at higher temperatures depends on the annealing temperature even less. Because of a very low roughness, distribution of hexagons is fairly uniform for all temperatures; their sizes are uniform as well.

The sizes of graphene crystals increase significantly with temperature, showing an effective activation energy, $\mathrm{E}_{\mathrm{gr}}=5 \mathrm{eV}$. The opposite change of the nucleation density and hexagons' size produces coverage that does not change as significantly; it slightly increases with the growth temperatures. Note that the density of domains grown at high temperature $\left(\sim 2 \times 10^{3}\right.$ $\mathrm{mm}^{-2}$ in our case) is 7 orders of magnitude less than the calculated surface density of impurities $\left(\sim 2 \times 10^{10} \mathrm{~mm}^{-2}\right.$, based on $0.2 \%$ of impurity content $)$. This suggests that the majority of impurities do not likely to accumulate at the surface or that they play insignificant role in graphene nucleation. Because of the lower nucleation density, individual crystals can grow close to $1 \mathrm{~mm}$ edge-to-edge at temperatures approaching the melting point of copper catalyst and at reduced methane pressures. Even larger crystals could be obtained by starting the growth at lower initial carbon source partial pressure and gradually increasing it. [16,20]

\section{Low pressure CVD (LPCVD)}

Graphene growth under low pressure illustrates similar temperature trends (Figure 2) agreeing with previously published results.[23] The apparent activation energy for LPCVD is substantially lower than in APCVD, resulting in a less pronounced but still significant temperature dependence with $\mathrm{E}_{\text {nuc }} \sim 4 \mathrm{eV}$ in the same temperature range. Notably, it appears that the effective activation energy does not depend on the ratio of the precursors partial pressures similar $\sim 4 \mathrm{eV}$ was measured for $\mathrm{P}_{\mathrm{H} 2} / \mathrm{P}_{\mathrm{CH} 4}=50$ and 1800 , which suggests that this activation energy cannot be attributed to the hydrogen assisted dehydrogenation processes of carbon precursor but has other common underlying cause. However, the rates of active carbon production and thus the nucleation densities do depend on the individual partial pressures of methane and hydrogen with the $\mathrm{P}_{\mathrm{H} 2} / \mathrm{P}_{\mathrm{CH} 4}$ ratio being one of the defining factors. [20] For example, Figure 2 shows that the nucleation densities for $\mathrm{P}_{\mathrm{H} 2} / \mathrm{P}_{\mathrm{CH} 4}=50$ is almost two orders of magnitude less as compared to $\mathrm{P}_{\mathrm{H} 2} / \mathrm{P}_{\mathrm{CH} 4}=1800$.

\section{Influence of the copper crystallographic orientations.}

On the low side of the studied temperature range, $\mathrm{T}<1000{ }^{\circ} \mathrm{C}$, we also see inequalities of the nucleation densities on different crystallographic orientations. For example, Figure 3 shows EBSD map of a polycrystalline foil used in APCVD of graphene at $970{ }^{\circ} \mathrm{C}$ and SEM image of the same foil. Crystallographic orientation of the metal catalyst plays important role in graphene growth not only on copper,[24] but on other metals such as nickel.[6,24] Nucleation density at this temperature on the nominal (111) copper orientation is approximately 3 times higher compared to (100) and (101) orientations for the atmospheric conditions used (Figure 3).

Similar variations in the nucleation densities are also observed at $950{ }^{\circ} \mathrm{C}$ but at higher temperatures, $\mathrm{T} \geq 1000{ }^{\circ} \mathrm{C}$, there is no recognizable difference between the copper orientations, which is in part due to larger sizes of graphene crystals that become comparable to the size of 
copper domains. Similar lack of discrimination for graphene nucleation on different $\mathrm{Cu}$ surface orientations at temperatures above $1000{ }^{\circ} \mathrm{C}$ we observe in LPCVD. Again, the average domain size in LPCVD has the activation energy roughly half of that for the nucleation density, making the total coverage almost temperature independent.

\section{Discussion.}

Thus the activation energy of nucleation is not affected by the surface orientation or by the ratio between $\mathrm{H}_{2}$ and methane but it is dramatically different between APCVD and LPCVD. What processes are responsible for such large activation energies of nucleation in APCVD, and what makes the graphene growth in APCVD so different from LPCVD?

The graphene nucleation density depends on several core processes happening on the copper surface[18,19] including the obvious (i) gas precursor adsorption, (ii) formation of active carbon species (dehydrogenation), (iii) diffusion of active carbon on the surface, and (iv) critical size nuclei formation that competes with (v) desorption. Most of these processes are affected by the background pressure and thus can potentially be responsible for the observed difference between the low and atmospheric pressure growth conditions. Pressure dependence of the reaction enthalpies should not be of any noticeable importance here because, even at the atmospheric pressure, the deviations from the ideal gas behavior are minimal, at least for the methane and hydrogen concentrations used. Desorption of species from the catalyst surface, however, is known to have a very pronounced dependence on the overall pressure. Due to collisions with the buffer gas in the diffusion layer close to the surface, the desorbed species have a higher returning rate to the surface at higher pressures. The effect is inversely proportional to the background pressure, making the evaporation rates at atmospheric pressure more than 3 orders of the magnitude lower than at reduced pressures.[22] The effect of background pressure in ref 22 is demonstrated using tungsten, but all metals are expected to show similar inverse proportionality of evaporation rates vs background pressure.

Desorbed species may include different hydrocarbons and, consequently, substantially different desorption energies at low and atmospheric conditions. Although a possible direct effect of the inert buffer gas on the structure of the surface species or the critical nuclei size is unclear, this may not be completely ruled out and further studies are needed.

Most of the previous studies that described graphene nucleation considered only active carbon species desorption.[17,23] Reported calculations of the carbon atoms adsorption energies on a copper surface vary within 4.1 to $7.5 \mathrm{eV}$ range,[17,26-30] while the intermediate methane dehydrogenation products $\mathrm{CH}_{\mathrm{x}}$ have significantly smaller adsorption energies, decreasing roughly by $\sim 1 \mathrm{eV}$ per an additional hydrogen atom.[29]

Except for a few reports, almost no attention has been given to the fact that copper itself has quite high evaporation rates in vacuum approaching $4 \mu \mathrm{m} /$ hour at $1000{ }^{\circ} \mathrm{C} .[4,31]$ This fact is obvious to anybody involved in LPCVD of graphene on $\mathrm{Cu}$ as the metal deposits are clearly observed in the deposition chamber. Such copper evaporation can also promote desorption of carbon species adsorbed on top of the catalyst and thus lead to a lower nucleation density. The binding energies of copper atoms depend on the surface geometry and the reported in the literature calculations fall within the range of $2.3-3.5 \mathrm{eV},[32]$ which is close to the experimentally measured heat of sublimation of copper, $3.5 \mathrm{eV}$.[33] The reported in literature experimentally measured activation energy of nucleation, $E_{\text {пис }} \sim 3 \mathrm{eV}[23]$ at low pressures and $\mathrm{T}$ $>950{ }^{\circ} \mathrm{C}$ is a bit lower of what we measure in our LPCVD, $E_{\text {nuc }} \sim 4 \mathrm{eV}$. Nevertheless, both values of $E_{\text {пис }}$ are close to the heat of sublimation of copper. Thus it would be logical to attribute $E_{\text {пис }}$ in 
LPCVD to copper evaporation that also causes successive surface active carbon desorption rather than just a direct desorption of carbon species.

At higher pressures, sublimation of copper is suppressed, thus the higher activation energies found here for APCVD can be linked to either the increased activation energy for copper atoms desorption (escaping the diffusional layer) or to desorption energy of carbon clusters from the surface (Figure 4). Adsorption energies of single carbon atoms cannot account for the experimentally measured $9 \mathrm{eV}$, and consequently, the critical nuclei must exceed 2 carbons. In fact, the carbon dimerization reaction, $2 \mathrm{C}(\mathrm{s}) \rightarrow \mathrm{C}_{2}(\mathrm{~s})$, is exothermic, which points to its spontaneity on copper surfaces, $[28,34,35]$ with consequent agglomeration and growth into larger carbon clusters.

According to DFT calculations,[36] the binding energy of a monolayer graphene on copper falls within the range $0.011-0.025 \mathrm{eV} / \AA^{2}$. Taking these values as a reference, a simple estimate for the critical size of surviving carbon cluster from $\mathrm{E}_{\text {nuc }} \sim 9 \mathrm{eV}$ can be made as $140-$ 320 atoms. This estimation is very crude given the fact that the carbon atoms at the edges may have significantly larger binding energies to copper compared to fully hybridized carbons in the graphene lattice.

Differences in the nucleation densities on various crystallographic orientations of copper have been reported previously[17,37,38] and they reflect an intricate interplay of various processes. Growth rates under LPCVD conditions were reported to have contradictory outcome. Deposition at $1000{ }^{\circ} \mathrm{C}$ by one group appears to have similar to the reported here preference for growth on (111) surface,[37] while deposition at $860{ }^{\circ} \mathrm{C}$ (that is below our temperature range) reported by another group to have (100) orientation coated by graphene faster than (111).[17] Theoretical calculations suggest that complete methane dehydrogenation on (111) copper surface has $\sim 1 \mathrm{eV}$ higher energy barrier as compared to the (100) orientation.[26] The adsorption energies of both, copper adatoms[32] and carbon monomers,[17] are lower on (111) and consequently should have lower activation energies for surface diffusion. All these effects contribute to the variations of the nucleation densities on different surface orientations at lower growth temperatures[17] and require further elucidation. At temperatures greater than $1000{ }^{\circ} \mathrm{C}$ these differences seem to be minimal for both APCVD and LPCVD. Nevertheless, suppressed desorption in APCVD is likely responsible for larger nucleation densities on (111) (Figure 3) that allows utilization for a stronger interaction between larger carbon clusters and the surface due to a better lattice match than in other orientations. Such epitaxial relation [38] between small carbon clusters and (111) copper helps in their stabilization and thus leads to higher nucleation densities that was observed for $\mathrm{T}<1000^{\circ} \mathrm{C}$.

We emphasize that the domains of graphene represent monolayers since they show homogeneous contrast in SEM [20] and the characteristic for monolayer $\mathrm{I}_{2 \mathrm{D}} / \mathrm{I}_{\mathrm{G}}>2$ ratio in the Raman spectra (Figure 3b). LEED measurements confirm the single crystal quality of the hexagonally shaped domains (Figure 5). Each single hexagonal domain shows exactly the same diffraction pattern at its different regions, as shown in Figure 5a-5d for one such grain, where the neighboring graphene grain shows a rotated diffraction pattern due to different orientation on the surface (e.g., Figure 5e). A faint black line, marked by white arrows on the LEEM image, indicates an apparent grain boundary of the copper separating the two grains. The fact that the LEED patterns are invariant over the graphene grains also indicates that the surface is relatively flat over this length scale. The single layer nature of these graphene domains can be also confirmed by the characteristic electron reflectivity data as discussed in detail elsewhere. [39,40] 
Thus we see that high deposition temperatures promote growth of large single crystal domains of graphene by minimizing the nucleation density. It is obviously not the only factor. While both the low and ambient pressure CVD methods afford good quality graphene samples, the choice of either one for large graphene domain production is also significantly affected by the technological aspects. Lower $\mathrm{E}_{\text {nuc }}$ in the LPCVD process may seem advantageous for large domain growth at temperatures much lower than copper melting but at AP conditions, similarly low nucleation densities can be obtained by decreasing the pre-exponential factor through varying the individual partial pressures $\mathrm{P}_{\mathrm{H} 2}$ and $\mathrm{P}_{\mathrm{CH} 4}$. The ratio between the latter changes the growth and etching rates and the resulting shape of the grains [20] but apparently not the activation energy for nucleation.

Larger graphene domains in APCVD can be obtained by gradual increase of the partial pressure of methane following the same strategy described previously.[20] For instance, Figure 6a shows $0.6 \mathrm{~mm}$ graphene crystal grown at $1080{ }^{\circ} \mathrm{C}$ by gradual increase of the methane concentration from 8 to $18 \mathrm{ppm}$ in a course of 3 hours while keeping the same $\mathrm{P}_{\mathrm{H} 2}=19$ Torr. The final domain has a star-like shape rather than a perfect hexagon, which points to a diffusion limited growth regime. Modeling of such star-like domain growth is illustrated in Figure 6b. We solved the diffusion equation, where the growth rate of graphene edges at every point was calculated as proportional to the local concentration gradient of the active species. The growing edges of graphene were described by the boundary condition of assigning the active species concentration to be zero. Figure $6 \mathrm{~b}$ also shows the lines of equal concentration gradient, which illustrate that the concentration gradient is higher at the corners, where the graphene crystal grows faster, resulting in a star-like shape.

Alternatively, decreasing the hydrogen pressure from the conditions of Figure 1 should also increase the $\mathrm{P}_{\mathrm{H} 2} / \mathrm{P}_{\mathrm{CH} 4}$ ratio and, correspondingly, the growth rate. In Figure 7 it was chosen $\mathrm{P}_{\mathrm{H} 2} / \mathrm{P}_{\mathrm{CH} 4} \sim 200$, which sustains close to hexagonal shape of domains. Further decrease of the $\mathrm{P}_{\mathrm{H} 2} / \mathrm{P}_{\mathrm{CH} 4}$ ratio by lowering the hydrogen pressure results in a more significant altering of the hexagonal shape. Even under conditions of Figure 7, hexagons begin to show development of dendritic edges, which nevertheless, do not point to any flaws in their single crystal structure. The hexagonal appearance is easier to interpret as single crystals over a variety of other shapes which may or may not be single crystals and require laborious characterization by Raman, LEEM or TEM. Note that mere decrease of the $\mathrm{P}_{\mathrm{H} 2} / \mathrm{P}_{\mathrm{CH} 4}$ ratio was only partially effective in increasing the graphene domain size (at least for the range of pressures used) compared to high hydrogen pressures (Figure 1).

Finally, from the technological perspective, APCVD emerges as a more convenient choice for mass production of high quality graphene as compared to LPCVD because it requires less equipment and in which evaporation of copper in the reactor [41,42] is practically eliminated. Much lower diffusion coefficients of gases under the atmospheric pressure conditions introduces significant precursor concentration gradient along the reactor tube that requires special attention when graphene is grown on very large substrates. Nevertheless, as we have demonstrated, the approach with gradual increase of precursor concentration can be scaled up and high quality monolayer graphene sheets of up to $40 \mathrm{in}$. in size can be produced and transferred onto a polymeric substrate. [4]

\section{Conclusions.}

We have illustrated the importance of the background pressure in CVD synthesis of graphene on copper catalyst. Namely, we demonstrate that the activation energy for nucleation in 
atmospheric pressure CVD $(9 \mathrm{eV})$ is dramatically larger compared to the low pressure conditions value $(4 \mathrm{eV})$. We attribute the difference to a greater importance of copper evaporation under low background pressure, where severe copper evaporation dictates the desorption rate of active carbon from the surface, while at atmospheric pressure, the measured $9 \mathrm{eV}$ activation energy reveals its true cause -- the desorption energy of carbon clusters from the surface. We also see that the discrimination between different crystallographic surface orientations for both AP and LP CVD is more pronounced at lower deposition temperatures, $\mathrm{T}<1000{ }^{\circ} \mathrm{C}$. A greater nucleation density on (111) orientation of copper in such conditions is attributed to the described in literature better graphene epitaxy on that surface. At higher temperatures $\mathrm{T}>1000{ }^{\circ} \mathrm{C}$, the discrimination between different surface orientations reduces for both AP and LP CVD.

At any pressure conditions, the highest possible temperature should be used for synthesis of large single crystal graphene. Nearly millimeter $(0.6 \mathrm{~mm})$ hexagonal graphene domains were achieved here at $1080^{\circ} \mathrm{C}$ and their single crystal appearance was confirmed by LEED.

\section{Experimental Section}

Before graphene growth, $125 \mu \mathrm{m}$ thick copper foils $(99.8 \%$ purity, Nimrod Hall) were cleaned with acetone, IPA and electropolished in $\mathrm{H}_{3} \mathrm{PO}_{4}$ following the procedure published previously.[4] CVD was performed in a single zone 3 in. split furnace; samples were taken out at room temperature to avoid oxidation. $[4,43]$.

APCVD: Partial pressures of methane and hydrogen were controlled by mixing stock gas mixtures, $2.5 \%$ of $\mathrm{H}_{2}$ in $\mathrm{Ar}$ and $0.1 \%$ of $\mathrm{CH}_{4}$ in $\mathrm{Ar}$, in the desired ratio by altering the flow rates. The total flow was kept $500 \mathrm{sccm}$, most of which was from the $\mathrm{H}_{2} /$ Ar mixture, corresponding to 19 Torr of $\mathrm{H}_{2}$ partial pressure. Prior to deposition, the samples were typically annealed at the temperature of deposition for $30 \mathrm{~min}$ in the hydrogen/Ar mixture. The flow rate of $\mathrm{CH}_{4} / \mathrm{Ar}$ mixture during deposition was chosen for the desired amount of methane and never exceeded 40 sccm (i.e., no more than $60 \mathrm{mTorr}$ of methane). Majority of the experiments were performed with 19 Torr of $\mathrm{H}_{2}$ and 10.5 mTorr of $\mathrm{CH}_{4}$ making $\mathrm{P}_{\mathrm{H} 2} / \mathrm{P}_{\mathrm{CH} 4}$ ratio equal to 1800 . Cooling was done under the flow of the hydrogen mixture by opening the split furnace, which corresponded to the initial rate exceeding $50{ }^{\circ} \mathrm{C} / \mathrm{min}$.

LPCVD: Two formulations were used to achieve different ratios of $\mathrm{P}_{\mathrm{H} 2} / \mathrm{P}_{\mathrm{CH} 4}$. In the first one, the total pressure was kept at 5 Torr during the whole cycle. Similar to APCVD, the samples were first annealed at the temperature of deposition for $30 \mathrm{~min}$ in pure hydrogen $(130 \mathrm{sccm})$. The growth was performed with $130 \mathrm{sccm}$ of pure $\mathrm{H}_{2}$ and using $730 \mathrm{sccm}$ of $100 \mathrm{ppm} \mathrm{CH}_{4}$ in $\mathrm{Ar}$ as a carbon source. Thus, the $\mathrm{P}_{\mathrm{H} 2} / \mathrm{P}_{\mathrm{CH} 4}$ ratio was matching that in APCVD, i.e. $\mathrm{P}_{\mathrm{H} 2} / \mathrm{P}_{\mathrm{CH} 4}=1800$. The data were collected for $75 \mathrm{~min}$ of deposition. In the second formulation, the total pressure was kept at 0.2 Torr and the growth was performed with $15 \mathrm{sccm}$ of pure $\mathrm{H}_{2}$ and $0.3 \mathrm{sccm}$ of pure $\mathrm{CH}_{4}$ to have $\mathrm{P}_{\mathrm{H} 2} / \mathrm{P}_{\mathrm{CH} 4}=50$. The data were collected after $30 \mathrm{~min}$ of deposition in this case. Cooling in both cases was also done under the flow of hydrogen with the initial rate exceeding $50{ }^{\circ} \mathrm{C} / \mathrm{min}$. The LPCVD experiments were performed after APCVD so that copper coating of the quartz tube introduced during LPCVD because of copper evaporation did not affect the otherwise clean conditions of APCVD.

Domain growth modeling. The domain growth simulation was performed using COMSOL 3.5a and Matlab software. The diffusion equation was evaluated using quasi equilibrium approach: after obtaining a steady state solution, each point was moved in the direction of the normal vector. The length of displacement was made proportional to the concentration gradient at each point with the scaling factor small enough to ensure negligible 
hexagon size increase after each step. For example, the shape change shown in Figure 5B required 50 calculation steps described above. The boundary conditions for the surface concentration of free carboneous species were: zero at crystal edges and unity at distances 100 times larger the size of the crystal. Each side of the hexagon was represented by 20 points with the corresponding segments between them.

Characterization. Low-energy electron microscope (LEEM) images together with lowenergy electron diffraction (LEED) patterns were acquired with an Elmitec LEEM III. The samples were briefly outgassed at $250^{\circ} \mathrm{C}$ in the instrument prior to measurements. EBSD were taken using a JEOL J6500F field-emission SEM equipped with EDAX-TSL Hikari I EBSD camera and OIM v5.31 software. The presence of graphene had only a slight influence on the copper EBSD pattern quality, and no effect on indexed orientation. Raman spectra were taken on Renishaw instrument with $633 \mathrm{~nm}$ excitation.

(a) Same scale
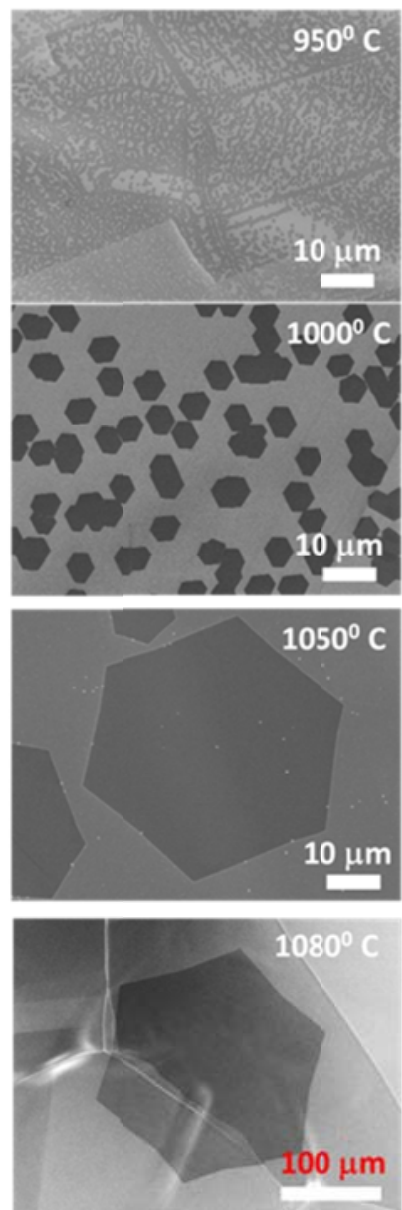

(b) Different Zoom
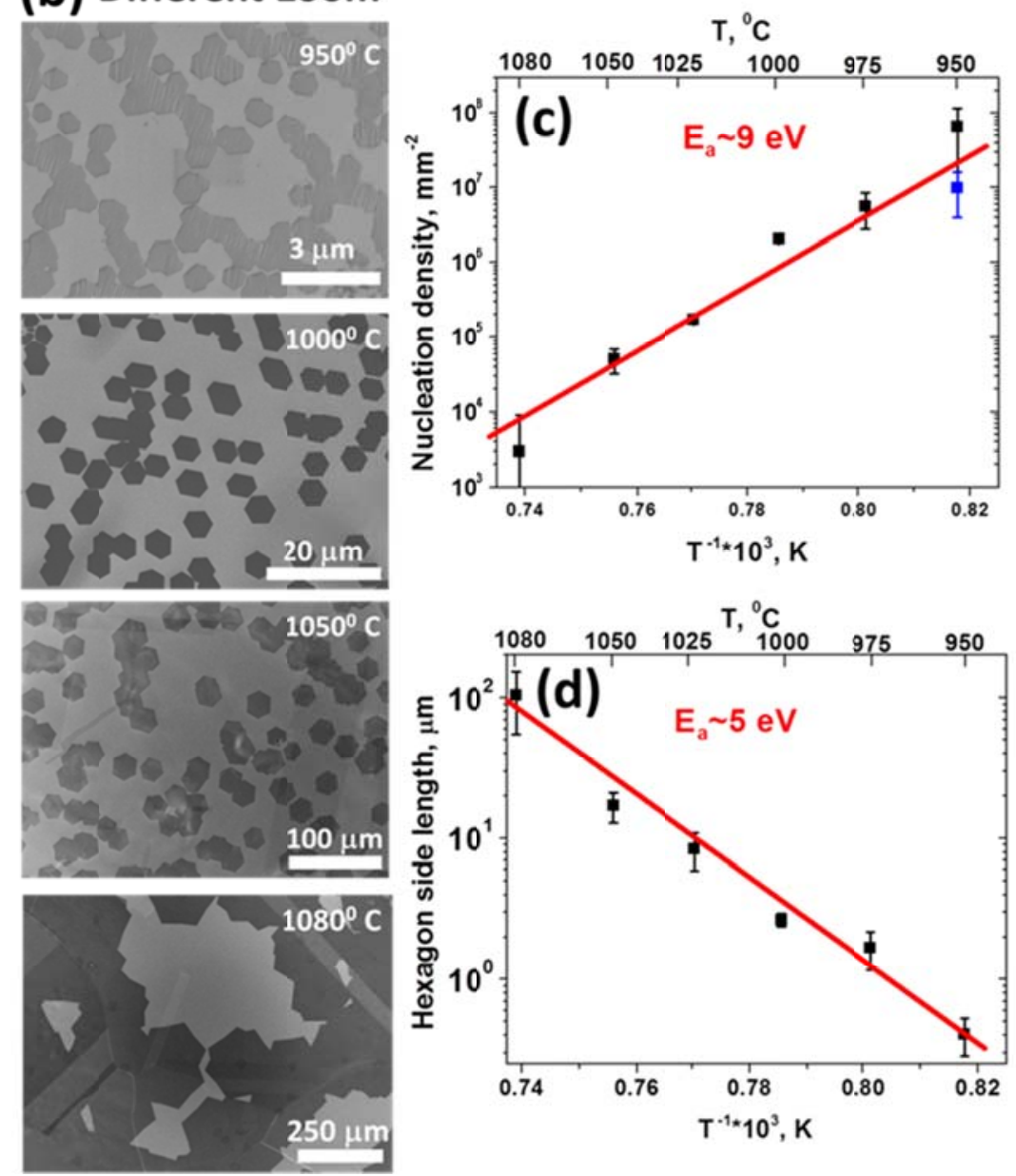

Figure 1. Temperature dependence of the nucleation density and graphene domain sizes for atmospheric pressure CVD: $P_{\mathrm{H} 2} / \mathrm{P}_{\mathrm{CH} 4}=1800$ (a) SEM images of graphene grains grown at different temperatures for constant partial pressures of $\mathrm{H}_{2}$ (19 Torr) and $\mathrm{CH}_{4}$ (10.5 mTorr). (b). Same as in (a), different zoom. (c) Arrhenius plot for the nucleation density. Blue point shows nucleation density at $950^{\circ} \mathrm{C}$, for sample annealed at $1080^{\circ} \mathrm{C}$. (d) Arrhenius plot for the grain size. 

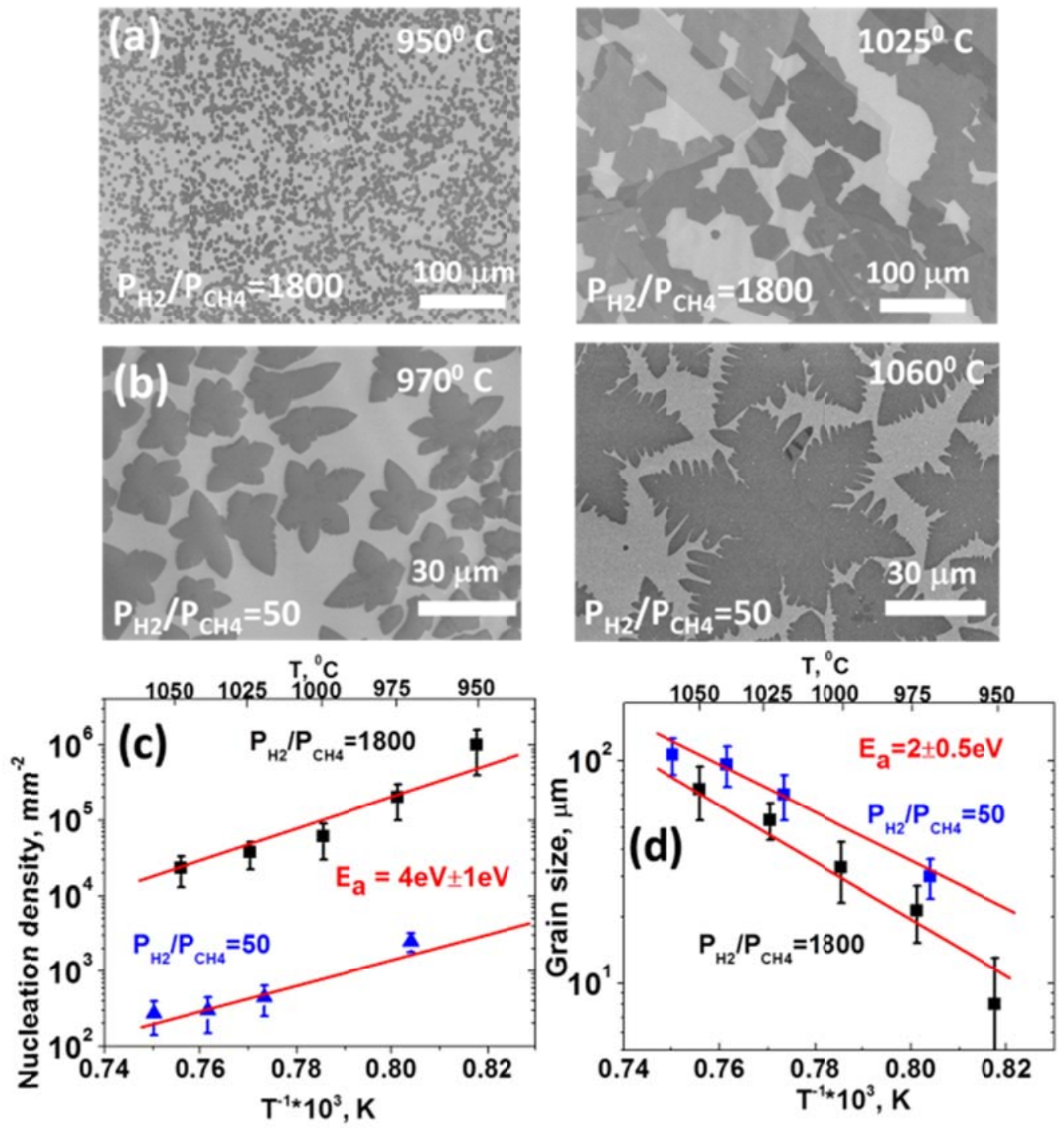

Figure 2. Temperature dependence of the nucleation density and domain sizes for low pressure CVD: (a) SEM images of hexagonal graphene grains grown at different temperatures keeping $P_{\mathrm{H} 2} / P_{\mathrm{CH} 4}=1800$. (b) Dendric/flower-like grain shapes for growth at lower hydrogen concentration: $P_{\mathrm{H} 2} / P_{\mathrm{CH} 4}=50$. Arrhenius plots for nucleation densities (c) and grain sizes (d). 

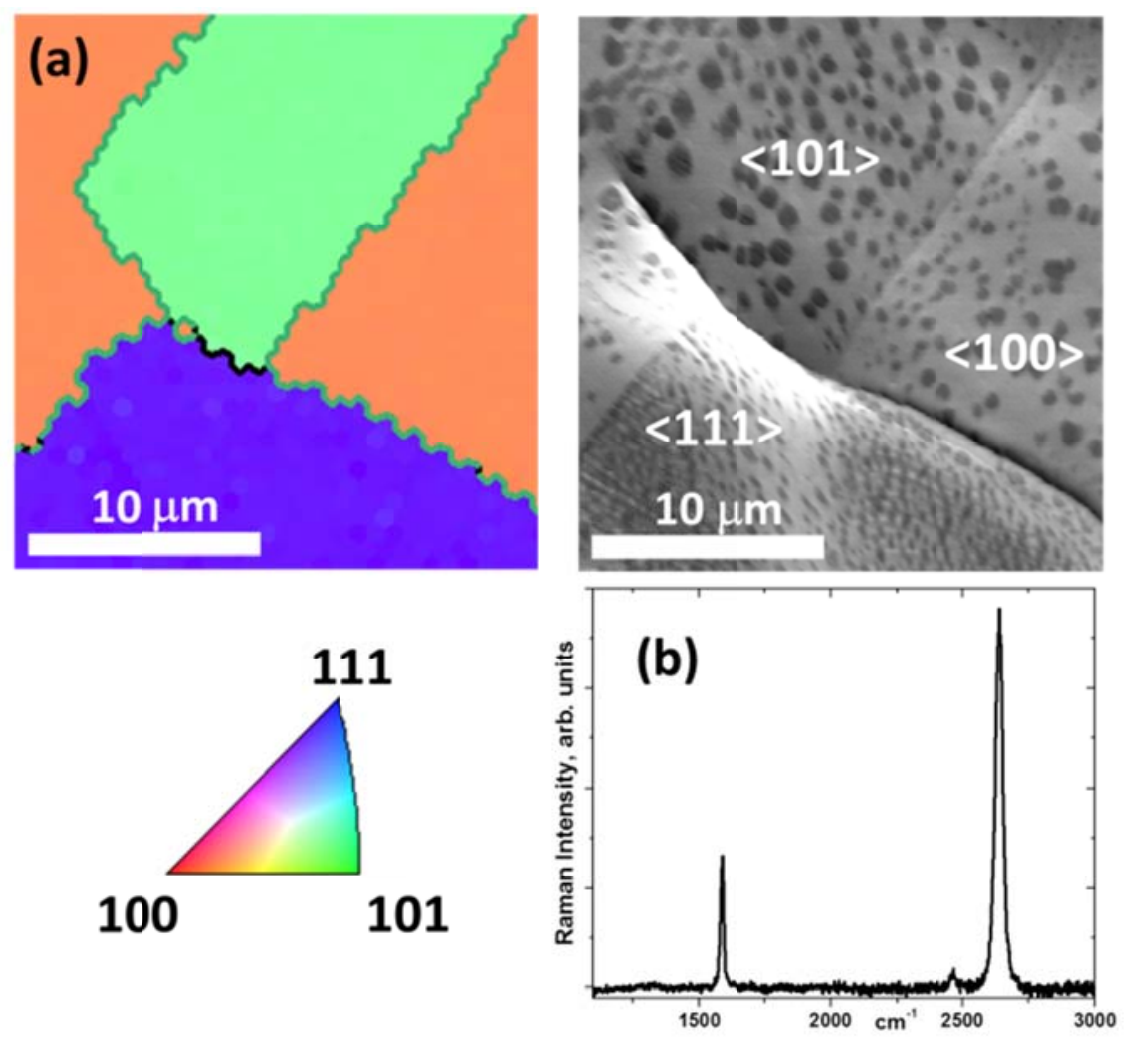

Figure 3. (a) Dependence of the graphene nucleation densities on the copper crystallographic orientations. Color scale indicates direction out of the page. Nucleation on (111) copper surface is approximately 3 times higher compare to (100) and (101). (b) Typical Raman spectrum taken in the middle of graphene grain.

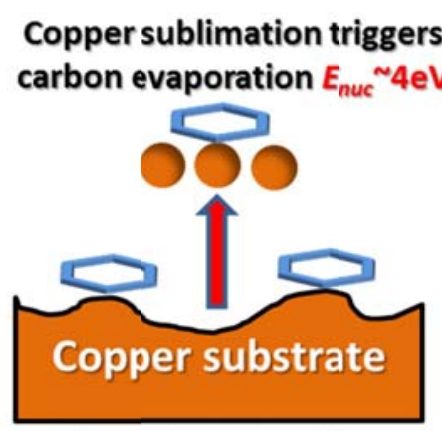

Low Pressure CVD

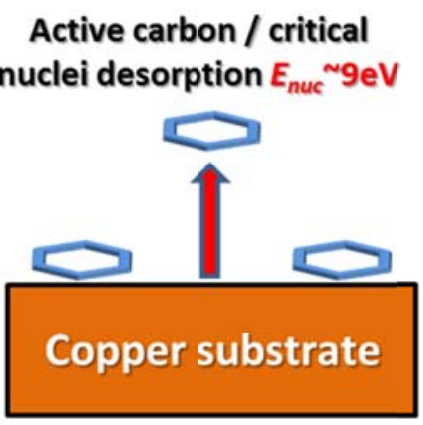

Atmospheric Pressure CVD

Figure 4. Activation energies differ for low and atmospheric pressure CVD. At low pressure evaporation of copper is significant and limits carbon nucleation (left) with $\mathrm{E}_{\text {nuc }} \sim 4 \mathrm{eV}$. Copper evaporation is suppressed at atmospheric pressure CVD (right) and the measured $\mathrm{E}_{\mathrm{nuc}} \sim 9 \mathrm{eV}$ is attributed to desorption energies of active carbon species / graphene critical nuclei. 

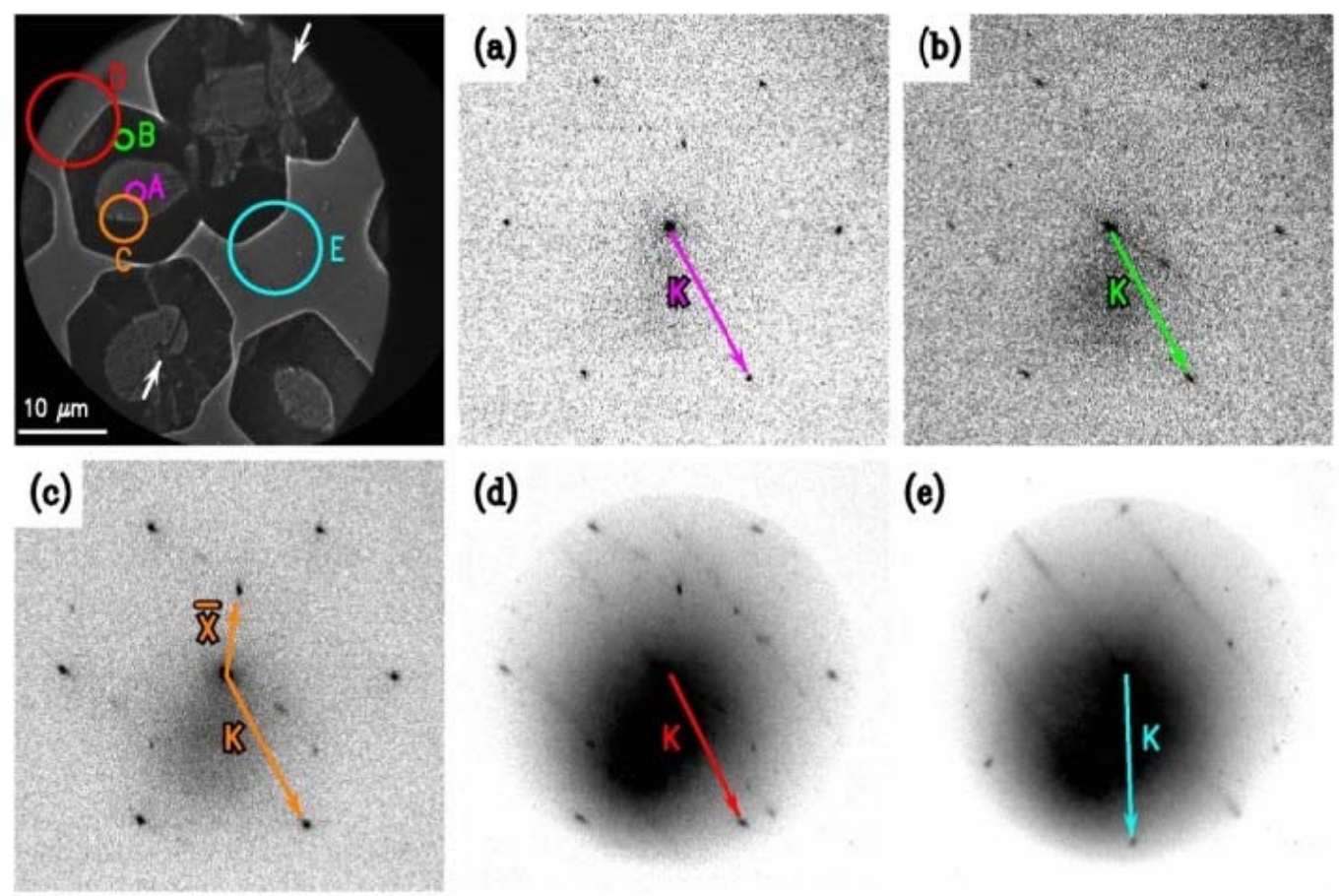

(d)

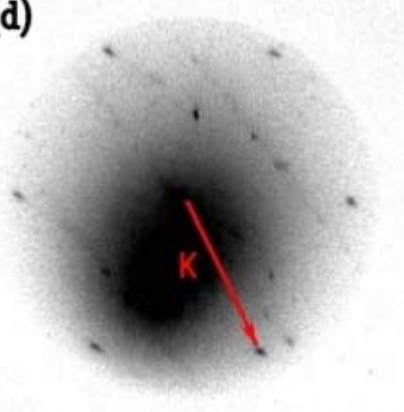

(e)

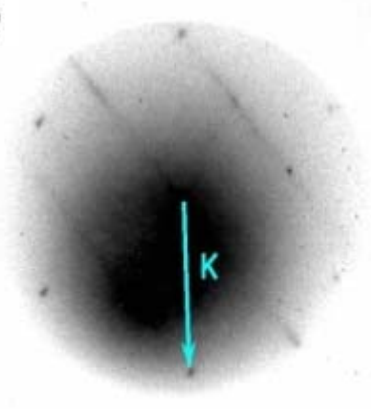

Figure 5. LEED confirms the single crystal appearance of hexagons. A LEEM image (acquired at $1.5 \mathrm{eV}$ ) is displayed in the upper left, showing hexagonal shaped graphene grains. LEED patterns $($ at $44 \mathrm{eV})$ were acquired at the locations $\mathrm{A}-\mathrm{E}$, over areas equal in size to the circles shown. These LEED patterns are displayed in (a) - (e), respectively. The patterns (a) - (d) taken on the same graphene grain all show identical orientation, whereas pattern (e) taken on a neighboring grain shows a rotated orientation. The K-point diffraction spot from graphene is indicated in each pattern. Some patterns also show a spot from the (100)-oriented $\mathrm{Cu}$ substrate ( $\overline{\mathrm{x}}$-point, indicated in pattern (c)), and double-diffraction leads to replication of certain graphene spots by this wavevector. Larger acquisition areas for the LEED patterns leads to significant secondary electron signal, forming a diffuse background (broad black region) as prominently seen in patterns (d) and (e).
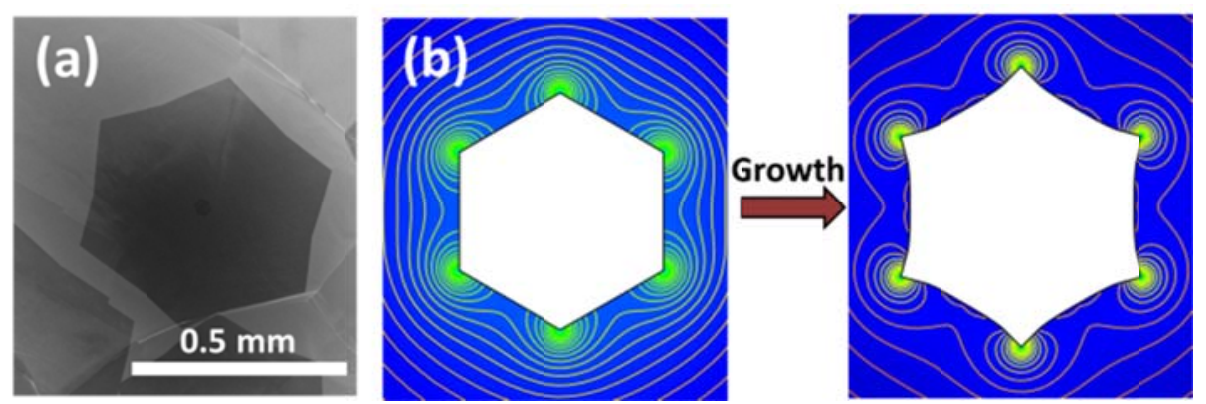

Figure 6. Diffusion limited growth: large star-shaped graphene crystals. (a) Large star-shaped hexagonal crystals were synthesized at temperatures approaching melting point of copper, $\mathrm{T}=$ $1080^{\circ} \mathrm{C}$. (b) Modeling of the growth: experimentally observed star-like graphene crystal shape is determined by the diffusion limited growth regime. Lines show equal concentration gradient boundaries. Modeling was done by solving diffusion equation with the growth rate proportional to the concentration gradient on crystal edges. Since the concentration gradient is higher at the corners, crystal grows faster in these directions. See text for details. 


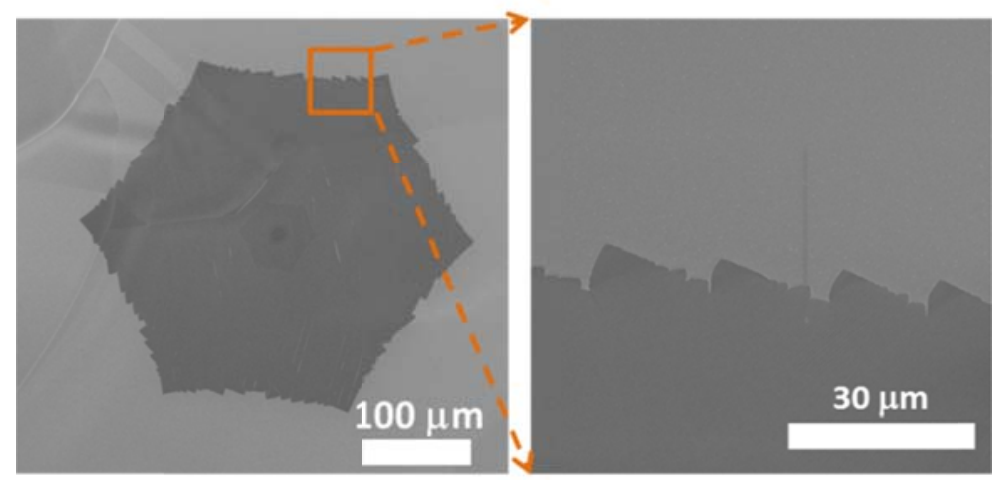

Figure 7. Growth at lower $\mathrm{H}_{2}$ partial pressure. Lowering hydrogen concentration (5 Torr of $\mathrm{H}_{2}$ instead of 19 Torr used above) and increase of methane to 26 mTorr results in a faster growth and yields dendritic domain edges yet has hexagonal shape pointing to the flawless single crystal appearance.

\section{ACKNOWLEDGMENT}

The LEEM/LEED work at CMU was supported by the National Science Foundation, grant DMR-0856240. A portion of this research was conducted at the Center for Nanophase Materials Sciences, which is sponsored at Oak Ridge National Laboratory by the Scientific User Facilities Division, Office of Basic Energy Sciences, U.S. Department of Energy and at the Oak Ridge National Laboratory's Shared Research Equipment (ShaRE) User Program, which is sponsored by the Office of Basic Energy Sciences, U.S. Department of Energy.

1. Li, X.; Cai, W.; An, J.; Kim, S.; Nah, J.; Yang, D.; Piner, R.; Velamakanni, A.; Jung, I.; Tutuc, E.; et al. Large-Area Synthesis of High-Quality and Uniform Graphene Films on Copper Foils. Science 2009, 324, 1312-1314.

2. Bae, S.; Kim, H.; Lee, Y.; Xu, X.; Park, J.-S.; Zheng, Y.; Balakrishnan, J.; Lei, T.; Kim, H. R.; Song, Y. et al. Roll-to-roll Production of 30-inch Graphene Films for Transparent Electrodes, Nat. Nanotech., 2010, 5, 574

3. Han, G.H.; Shin, H.-J.; Kim E.S.; Chae S. J.; Choi, J.-Y.; Lee, Y.H. Poly(ethylene covinyl acetate)-assisted one-step transfer of ultra-large graphene, NANO, 2011, 6, 59

4. Vlassiouk, I.; Fulvio, P.; Meyer, H.; Lavrik, N.; Dai, S.; Datskos, P.; Smirnov, S. Large Scale Atmospheric Pressure Chemical Vapor Deposition of Graphene and references therein, Carbon, 2013, 54, 58.

5. Huang, P. Y.; Ruiz-Vargas, C. S.; van der Zande, A. M.; Whitney, W. S.; Levendorf, M.; Kevek, J.; Garg, S.; Alden, J. S.; Hustedt, C. J.; Zhu, Y. et al. Grains and Grain Boundaries in Single-layer Graphene Atomic Patchwork Quilts, Nature, 2011, 469, 389

6. Zhang, Y.; Gao, T.; Xie, S.; Dai, B.; Fu, L.; Gao, Y.; Chen, Y.; Liu, M.; Liu, Z.; Different Growth Behaviors of Ambient Pressure Chemical Vapor Deposition Graphene on Ni(111) and Ni Films: A Scanning Tunneling Microscopy Study, Nano Research, 2012, 5, 402

7. Vlassiouk, I.; Smirnov, S.; Ivanov, I.; Fulvio, P.; Dai, S.; Meyer, H.; Chi, M.; Hensley, D.; Datskos, P.; Lavrik, N. Electrical and Thermal Conductivity of Low Temperature CVD Graphene: the Effect of Disorder, Nanotechnology, 2011, 22, 275716 
8. Yan, Z.; Lin, J.;Peng, Z.; Sun, Z; Zhu, Y.; Li, L.; Xiang, C.; Samuel, E.L.; Kittrell, C.; Tour, L.M. Toward the Synthesis of Wafer-Scale Single-Crystal Graphene on Copper Foils, ACS Nano, 2012, 6, 9110

9. Wu, Y.A.; Fan, Y.; Speller, S.; Creeth, G.L; Sadowski,J.T.; He, K.; Robertson, A.W.; Allen, C.S.; Warner, J.H. Large Single Crystals of Graphene on Melted Copper Using Chemical Vapor Deposition, ACS Nano, 2012, 6, 5010

10. Geng, D.; Wu, B.; Guo, Y.;Huang, L.; Xue, Y.; Chen, J.; Yu, G.; Jiang, L.; Hu, W.; Liu, Y. Uniform hexagonal graphene flakes and films grown on liquid copper surface, PNAS, 2012, 109, 7992

11. Wang, H.; Wang, G.; Bao, P.; Yang, S.; Zhu, W.; Xie, X.; Zhang, W.J. Controllable Synthesis of Submillimeter Single-Crystal Monolayer Graphene Domains on Copper Foils by Suppressing Nucleation, J. Am. Chem. Soc., 2012, 134, 3627

12. Wu, T.; Ding, G.; Shen, H.; Wang, H.; Sun, L.; Jiang, D.; Xie, X.; Jiang, M. Triggering the Continuous Growth of Graphene Toward Millimeter-Sized Grains, Adv. Funct. Mat., 2013, 23,198

13. Li, X.; Magnuson, C. W.; Venugopal, A.; Tromp, R. M.;Hannon, J. B.; Vogel, E. M.; Colombo, L.; Ruoff, R. S. Large-Area Graphene Single Crystals Grown by Low-Pressure Chemical Vapor Deposition of Methane on Copper, J. Am. Chem. Soc., 2011, 133, 2816

14. Gao, L.; Ren, W.; Xu, H.; Jin, L.; Wang, Z.; Ma, T.; Ma, L.-P.; Zhang, Z.; Fu, Q.; Peng, L.-M.; Bao, X.; Cheng, H.-M. Repeated growth and bubbling transfer of graphene with millimetre-size single-crystal grains using platinum, Nat. Comm., 2012, 3, 699

15. Ago, H.; Ogawa, Y.; Tsuji, M.; Mizuno, S.; Hibino, H. "Catalytic Growth of Graphene: Toward Large-Area Single-Crystalline Graphene", Phys. Chem. Lett., 2012, 3, 2228

16. Li, X.; Magnuson, C. W.; Venugopal, A.; An, J.; Suk, J. W.; Han, B.; Borysiak, M.; Cai, W.; Velamakanni, A.; Zhu, Y. et al. Graphene Films with Large Domain Size by a Two-Step Chemical Vapor Deposition Process, Nano Lett., 2010, 10, 4328-4334.

17. Hayashi, K.; Sato, S.; Ikeda, M.; Kaneta, C.; Yokoyama, N. "Selective Graphene Formation on Copper Twin Crystals", J. Am. Chem. Soc., 2012, 134, 12492

18. Venables, J.A.; Spiller G.D.T.; Hanbucken, M. Nucleation and growth of thin films, Rep. Prog. Phys., 1984, 47, 399

19. Robinson, V.N.E. and Robins, J. L. Nucleation Kinetics of Gold Deposited Onto UHV Cleaved Surfaces of $\mathrm{NaCl}$ and $\mathrm{KBr}$, Thin Solid Films, 1974, 20, 155

20. Vlassiouk, I.; Regmi, M.; Fulvio, P.; Dai, S.; Datskos, P.; Eres, G.; Smirnov, S., Role of Hydrogen in Chemical Vapor Deposition Growth of Large Single-Crystal Graphene. ACS Nano 2011, 5 (7), 6069-6076

21. V. I. Shapovalov, Metal-Hydrogen Phase Diagrams in the Vicinity of Melting Temperatures, Proceedings of the 1999 International symposium on Liquid Metal Processing and Casting

22. Fonda, G.R. Evaporation of Tungsten Under Various Pressures of Argon, Phys. Rev., 1928, 31,260

23. Kim, H.; Mattevi, C.; Calvo, M.R.; Oberg, J.C.; Artiglia, L.; Agnoli, S.; Hirjibehedin, C.F.; Chhowalla, M.; Saiz, E. Activation Energy Paths for Graphene Nucleation and Growth on $\mathrm{Cu}$, ACS Nano, 2012, 6, 3614

24. Wu, Y.; Robertson, A.W.; Schaffel, F.; Speller, S.C.;Warner, J.H. Aligned Rectangular Few-Layer Graphene Domains on Copper Surfaces, Chem. of Mater., 2011, 23, 4543 
25. Orofeo, C.M.; Ago, H.; Hu, B.; Tsuji, M. Synthesis of Large Area, Homogeneous, Single Layer Graphene Films by Annealing Amorphous Carbon on Co and Ni, Nano Research, 2011, 4, 531

26. Zhang, W.; Wu, P.; Li, Z.;Yang, J. First-Principles Thermodynamics of Graphene Growth on Cu Surfaces, J. Phys. Chem. C, 2011, 115, 17782

27. Pang, X.-Y.; Xue, L.-Q.; Wang, G.-C. Adsorption of Atoms on Cu Surfaces: A Density Functional Theory Study, Langmuir, 2007, 23, 4910

28. Gajewski, G. and Pao, C. W. Ab initio calculations of the reaction pathways for methane decomposition over the $\mathrm{Cu}$ (111) surface, J. Chem. Phys., 2011, 135, 064707

29. Hei, M.J.;Chen, M.J;Yi, J.; Lin, Y.J.; Lin, Y.Z.; Wei, G.; Liao, D.W. CO2-reforming of methane on transitionmetalsurfaces, Surf. Science, 1998, 417, 82

30. Zeigarnik, A.V.; Valdes-Perez, R.E.; Myatkovskaya, O.N. C-C Bond Scission in Ethane Hydrogenolysis, J. Phys. Chem. B, 2000, 104, 10578

31. S. Chen, H. Ji, H. Chou, Q. Li, H. Li, J.W. Suk, R. Piner, L. Liao, W. Cai, R.S. Ruoff, Millimeter-Size Single-Crystal Graphene by Suppressing Evaporative Loss of $\mathrm{Cu}$ During Low Pressure Chemical Vapor Deposition, Adv. Mat., 2013, 25, 2062-2065

32. Karimi, M.; Tomkowski, T.; Vidali, G.; Biham, O. Diffusion of $\mathrm{Cu}$ on Cu Surfaces, Phys. Rev. B., 1995, 52, 5364

33. Hersh, H. N. The Vapor Pressure of Copper, J. Am. Chem. Soc.,1953, 75, 1529

34. Yazyev, O. and Pasquarello, A. Effect of Metal Elements in Catalytic Growth of Carbon Nanotubes, Phys. Rev. Lett., 2008, 100, 156102

35. Riikonen, A.; Krasheninnikov, A.V.; Halonen, L.; Nieminen, R.M. The Role of Stable and Mobile Carbon Adspecies in Copper-Promoted Graphene Growth, J. Phys. Chem. C., 2012, 116,5802

36. Xu, Z.; Buehler, M.J. Interface structure and mechanics between graphene and metal substrates: a first-principles study, Journal of Physics: Condensed Matter, 2010, 22, 485301

37. Wood, J.D.; Schmucker, S.W.; Lyons, A.S.; Pop, E.; Lyding, J.W. Effects of Polycrystalline $\mathrm{Cu}$ Substrate on Graphene Growth by Chemical Vapor Deposition. Nano Lett. 2011, 11, 4547.

38. Gao, L.; Guest, J. R.; Guisinger, N. P. Epitaxial Graphene on Cu(111). Nano Lett. 2010, 10,3512 .

39. Nie, S.; Wu, W.; Xing, S.; Yu, Q.; Bao, J.; Pei, S.-S.; and McCarty, K. F. Growth from Below: Bilayer Graphene on Copper by Chemical Vapor Deposition, New J. of Physics 2012, 14, 093028

40. Feenstra, R. M.; Srivastava, N.; Gao, Q.; Widom, M.; Diaconescu, B.; Ohta, T.; Kellogg, G. L.; Robinson, J. T.; Vlassiouk, I. V. Low-energy Electron Reflectivity from Graphene, Phys. Rev. B 2013, 87, 041406(R).

41. Robinson, Z. R.; Tyagi, P.; Mowll, T. R.; Ventrice, C. A., Jr. Argon-Assisted Growth of Epitaxial Graphene on $\mathrm{Cu}(111)$. Phys. Rev. B 2012, 235413.

42. $\quad$ Kidambi, P. R.; Ducati, C.; Dlubak, B.; Gardiner, D.; Weatherup, R. S.; Martin, M.-B.; Seneor, P.; Coles, H.; Hofmann, S. The Parameter Space of Graphene Chemical Vapor Deposition on Polycrystalline Cu. J. Phys. Chem. C 2012, 116, 22492-22501.

43. Surwade, S.; Li, Z.; Liu, H. Thermal Oxidation and Unwrinkling of Chemical Vapor Deposition-Grown Graphene. J. Phys. Chem. C 2012, 116, 20600-20606. 